\title{
Human chorionic gonadotropin $\beta$ regulates epithelial-mesenchymal transition and metastasis in human ovarian cancer
}

\author{
NA LIU ${ }^{1 *}$, SHU-MIN PENG ${ }^{2 *}$, GUANG-XI ZHAN ${ }^{2 *}$, JING YU $^{3}$, WEI-MIN WU ${ }^{1}$, \\ $\mathrm{HAO} \mathrm{GAO}^{1}$, XIAO-FENG $\mathrm{LI}^{1}$ and XIAO-QING GUO ${ }^{1}$ \\ ${ }^{1}$ Department of Obstetrics and Gynecology, Shanghai First Maternity and Infant Hospital, \\ Tongji University School of Medicine, Shanghai 200040; ${ }^{2}$ Department of Obstetrics and Gynecology, \\ The First Affiliated Hospital, Shihezi University School of Medicine, Shihezi, Xinjiang 832000; \\ ${ }^{3}$ Department of Pathology, Shanghai First Maternity and Infant Hospital, \\ Tongji University School of Medicine, Shanghai 200040, P.R. China
}

Received February 13, 2017; Accepted June 30, 2017

DOI: $10.3892 / o r .2017 .5818$

\begin{abstract}
Human chorionic gonadotropin $\beta$ ( $\beta$-hCG) is a wellknown and accurate marker for the diagnosis and monitoring of pregnancy, trophoblastic tumors and ovarian germ cell tumors. Recently, $\beta$-hCG has been found to be closely related to poor prognosis and metastasis in various other malignant tumors, while its role and mechanism in ovarian cancer is still unclear. In the present study, lentiviral-mediated transfection and small interfering RNA (siRNA) were used to alter $\beta$-hCG expression in the ovarian cancer cell lines ES-2 and SKOV3, respectively. Then, migration and invasion activity regulated by $\beta$-hCG were evaluated by wound-healing and Transwell assays in vitro and in a peritoneal xenograft nude mouse model in vivo. EDTA and trypsin were utilized to investigate the attachment ability of these cells. Moreover, the expression of epithelial mesenchymal transition (EMT) markers ( $\beta$-catenin, Slug, vimentin, Snail, claudin, E-cadherin and N-cadherin) was assessed by western blotting and immunofluorescence in ES- 2 and SKOV3 cells. Furthermore, $\beta$-hCG and EMT markers were evaluated in human ovarian cancer specimens by IHC. The results showed that overexpression of $\beta$-hCG clearly promoted migration and invasion in ES-2 and SKOV3 cells $(\mathrm{P}<0.05)$ and facilitated metastasis in peritoneal xenografts, while silencing of $\beta$-hCG led to the opposite effect. Moreover, $\beta$-hCG was closely associated with cell morphology, attachment ability and EMT marker expression in ES-2 and SKOV3
\end{abstract}

Correspondence to: Professor Xiao-Qing Guo, Department of Obstetrics and Gynecology, Shanghai First Maternity and Infant Hospital, Tongji University School of Medicine, 536 Changle Road, Shanghai 200040, P.R. China

E-mail: xiaoqing_guo@163.com

*Contributed equally

Key words: ovarian cancer, $\beta$-hCG, metastasis, EMT cells and human ovarian cancer specimens. Upregulation of $\beta$-hCG promoted cells from an epithelial-like morphology to a mesenchymal-like phenotype, decreased the adhesion ability $(\mathrm{P}<0.05)$, and reduced the expression of epithelial markers (E-cadherin) while inducing the expression of mesenchymal markers (vimentin, $\mathrm{N}$-cadherin, $\beta$-catenin and Slug). Furthermore, the converse effects were confirmed by knockdown of $\beta$-hCG. These findings strongly suggest that $\beta$-hCG may regulate metastasis of ovarian cancer through EMT, and it may become a new target for therapeutic intervention.

\section{Introduction}

Ovarian carcinoma is the most lethal gynecologic malignancy (1). To date, there is no effective early screening method, and more than one-half of cases are diagnosed at an advanced stage (2). Unlike other gynecological malignancies, extensive pelvic and peritoneal dissemination is the most common path of metastasis in ovarian cancer (3). Therefore, better understanding of the mechanisms of cell migration and invasion is urgently needed to identify novel therapeutic strategies for ovarian cancer treatment.

Human chorionic gonadotropin (hCG) has a physiologically significant role during pregnancy. The hCG family refers to a group of five molecules, each sharing a common amino acid sequence but differing in multimeric structure and carbohydrate side chain structure. Of these five molecules, $\beta$-hCG and hyperglycosylated $\beta$-hCG were confirmed to be associated with advanced malignancies (4). Recently, it was shown that elevated levels of $\beta$-hCG in serum, urine or tumor tissue correlates with poor prognosis, aggressiveness and resistance to therapy in a variety of nontrophoblastic tumors, such as bladder, colon, lung (5) and testicular cancer (6). Moreover, it is also ectopically expressed in numerous gynecological malignancies, such as endometrial carcinoma (7), ovarian cancer $(8)$ and cervical carcinoma $(9,10)$.

In our previous study, $\beta$-hCG was confirmed to facilitate proliferation and cell cycle progression, attenuate apoptosis and 
promote tumorigenesis in ovarian surface epithelial cells (11). To test whether this molecule plays a potential role in ovarian epithelial cancer tumorigenesis, a series of experiments were performed. We found that the $\beta$-hCG level was significantly elevated in metastatic tissue compared to the level noted in the primary ovarian cancer tissue, which indicated that $\beta-\mathrm{hCG}$ may play a role in ovarian cancer metastasis. To further verify this hypothesis, a series of in vitro and in vivo investigations were performed.

\section{Materials and methods}

Patients and tissue specimens. The present study was approved by the Medical Ethics Committee at The First Affiliated Hospital of the Medical College of Shihezi University. Written consent was obtained from all enrolled patients. Paraffin-embedded human ovarian cancer specimens were obtained from 24 patients who underwent surgical resection without prior adjuvant therapy from December 2006 to December 2013 at The First Affiliated Hospital of the Medical College of Shihezi University, and 20 cases of normal ovarian tissue samples were used as a control. All samples were pathologically diagnosed according to the World Health Organization (WHO) classification guidelines (2004). All procedures were performed in accordance with the Declaration of Helsinki.

Cell culture. The human ovarian epithelial cancer cell lines ES-2 and SKOV3 used in the present study were obtained from the American Type Culture Collection (ATCC; Manassas, VA, USA). Cells were maintained in RPMI-1640 medium (HyClone, Logan, UT, USA), containing 10\% fetal bovine serum (FBS; Gibco, Grand Island, NY, USA), 100 U/ml penicillin and $100 \mathrm{mg} / \mathrm{ml}$ streptomycin. Cells were cultured at $37^{\circ} \mathrm{C}$ in a humidified $5 \% \mathrm{CO}_{2}$ environment.

Immunohistochemistry (IHC). Following formalin fixation and paraffin-embedding, the $4-\mu \mathrm{m}$ thick tissue sections were incubated with primary rabbit polyclonal antibodies against $\beta$-hCG (1:50; ab53087; Abcam, Cambridge, MA, USA) overnight at $4^{\circ} \mathrm{C}$, washed with phosphate-buffered saline (PBS), and then incubated with the secondary antibody for $1 \mathrm{~h}$ at $37^{\circ} \mathrm{C}$. Finally, the sections were stained with 3,3'-diaminobenzidine and then counterstained with hematoxylin. Images were obtained with a Nikon Eclipse TE2000 fluorescence microscope (Nikon, Tokyo, Japan). Stained tissues were classified according to staining intensity by two investigators. The extent of $\beta$-hCG staining in tissue cores was quantified using a four-tier grading system: $0, \leq 5 \%$ positive staining; $1,5-20 \%$ positive staining; $2,20-50 \%$ positive staining; and $3, \geq 50 \%$ positive staining. For statistical analysis, we divided cases into two groups: negative expression (with scores of 0 ) and positive expression (with scores of 1, 2 or 3) (11).

Establishment of $\beta$-hCG-overexpressing cell lines. We established $\beta$-hCG-overexpressing ovarian cancer cell lines in ES-2 and SKOV3 cells via lentivirus transfection. A lentiviral vector encoding $\beta$-hCG (LV- $\beta$-hCG) and a negative control vector (LV-vector) were purchased from Obio Technology (Shanghai, China), carrying an enhanced green fluorescent protein reporter gene, eGFP. For $\beta$-hCG exogenous overexpression, lentivirus containing $\mathrm{LV}-\beta-\mathrm{hCG}$ or the $\mathrm{LV}$-vector were transfected into ES-2 and SKOV3 cells using Polybrene $(5.0 \mu \mathrm{g} / \mathrm{ml})$ from Obio Technology, following the manufacturer's instructions. Medium containing puromycin $(0.2 \mathrm{mg} / \mathrm{ml})$ was used to select stably transduced cells. The cells were photographed with a fluorescence microscope. $\beta$-hCG upregulation efficiency was assessed using qPCR and a western blot assay.

$\beta$-hCG-siRNA transfection in ovarian cancer cells. ES-2 and SKOV3 cells were separately seeded in plates, and then transfected with $\beta$-hCG-siRNAs or nc-siRNA (100 nM; RiboBio, Guangzhou, China) using Lipofectamine 2000 (Invitrogen, Carlsbad, CA, USA) transfection reagent, following the manufacturer's protocol. Cells were harvested at 48-72 h post-transfection for future experiments. $\beta$-hCG knockdown efficiency was assessed using qPCR and a western blot assay.

Wound healing and Transwell assays. Wound healing assay: cells were seeded into 24-well plates and allowed to grow to $90-95 \%$ confluence. Similar sized wounds were introduced to a monolayer of cells using a sterile white pipette tip. The wounded monolayer of cells was washed three times with PBS to remove cell debris and then cultured. The speed of wound closure was monitored and photographed every $4 \mathrm{~h}$ until the wound filled. Transwell assay: $1.0 \times 10^{5}$ cells in $100 \mu \mathrm{l}$ of RPMI-1640 with $2 \%$ FBS were seeded into Transwell upper chambers (cat. 3422; Corning Inc., Corning, NY, USA) with or without pre-coated Matrigel matrix (cat. 356234; BD Biosciences, Franklin Lakes, NJ, USA), and $500 \mu \mathrm{l}$ of RPMI-1640 containing 10\% FBS was added into the lower chamber to serve as the chemoattractant. After 16-48 $\mathrm{h}$ of incubation, the cells that did not migrate or invade through the pores were carefully removed. Cells on the filters were fixed in $100 \%$ methanol followed by hematoxylin staining (BA4025; Baso Diagnostics, Inc., Zhuhai, China). The number of migrated cells were counted with an inverted microscope (magnification, x200; Nikon Eclipse), in 10 random fields/chamber. All experiments were performed in triplicate.

Colony-formation assay. For colony-formation assays, cells were plated into 6-well plates at a concentration of 150 cells/well and incubated for $\sim 2$ weeks. Then, colonies of cells were observed, fixed with $100 \%$ methanol and stained with hematoxylin.

Cell adhesion assay. Cells were seeded into 6-well plates until they reached $90-95 \%$ confluence, and then, the cell culture medium was removed, and cells were washed twice with PBS. After that, the mixture of trypsin (cat. 25200072; Gibco) and EDTA (cat. E8008; Sigma-Aldrich, St. Louis, MO, USA) in a ratio of 1:20 was added into the plates, and cell images were captured at $0,5,10$ and $20 \mathrm{~min}$, separately. The pre-experimental adhesion assay was firstly performed to determine the optimal time in ES-2 and SKOV3 cells, and the remaining cells were captured and counted at different time points. According to the results of pre-experiment (data not shown), ES-2 and SKOV 3 cells were captured at 5 and $20 \mathrm{~min}$ after replacing the cell culture medium with the mixture of trypsin and EDTA, respectively. Finally, the cells that remained adherent were 
counted, the residual cell adhesion proportion was calculated, and the residual cells were divided to initial cells in ES-2 and SKOV 3 cells at 5 or $20 \mathrm{~min}$, respectively.

Immunofluorescence staining. For immunofluorescence microscopy, the cells were seeded on a culture dish (cat. 801001; Nest Biotechnology, Rahway, NJ, USA) and incubated with a primary antibody against E-cadherin, $\mathrm{N}$-cadherin, Snail, vimentin, $\beta$-catenin (cat. 9782; Cell Signaling Technology, Beverly, MA, USA), followed by incubation with Alexa 488-conjugated secondary antibody (Sigma, St. Louis, MO, USA). Fluorescence staining for vimentin was visualized by confocal laser-scanning microscopy (FluoView FV1000; Olympus, Japan); DAPI (Sigma) was used to counterstain DNA.

RNA extraction and real-time RT-PCR. Total RNA from ES-2 and SKOV3 cells was isolated by TRIzol reagent (Invitrogen) according to the manufacturer's protocol. Reverse transcription reactions were performed with PrimeScript ${ }^{\mathrm{TM}} \mathrm{RT}$ Master Mix kit (Takara Bio, Inc., Shiga, Japan) according to the protocol. RT-PCR was performed on an Applied Biosystems StepOnePlus ${ }^{\mathrm{TM}}$ Real-Time PCR System using a SuperReal PreMix Plus (SYBR-Green) kit (Tiangen Biotech, Beijing, China). The PCR cycling program was run with an initial predenaturation step at $95^{\circ} \mathrm{C}$ for $15 \mathrm{~min}$, then with 45 cycles for amplification, at $95^{\circ} \mathrm{C}$ for $10 \mathrm{sec}$ and $60^{\circ} \mathrm{C}$ for $32 \mathrm{sec}$.

GAPDH was used as an internal reference. Each test was performed in triplicate, and the $2^{-\Delta \Delta \mathrm{Ct}}$ method was used to calculate the expression of mRNA in each of the cell lines. The primers used were: $\beta$-hCG forward, 5'-TCTGTGCCGGCTA CTGCCCC-3' and reverse, 5'-TTGGGACCCCCGCAGTC AGT-3'; GAPDH forward, 5'-ACAACTTTGGTATCGTGGA AGG-3' and reverse, 5'-GCCATCACGCCACAGTTTC-3'.

Western blotting. Total protein from cells and tissues was lysed in KeyGen Whole Cell Lysis Assay (cat. KGP250; KeyGen Biotech, Nanjing, China). Then, 20-50 $\mu$ g protein/sample from different cell lines or treatments was separated by SDS-PAGE and blotted onto polyvinylidene fluoride (PVDF) membranes that were blocked for $2 \mathrm{~h}$ at room temperature with 5\% BSA in TBS containing $0.05 \%$ Tween-20. The membranes were then incubated overnight at $4^{\circ} \mathrm{C}$ in BSA in TBS containing $0.05 \%$ Tween-20 and probed with mouse antibodies against $\beta$-actin (1:4,000; cat. 20010; Abmart, Arlington, MA, USA) or rabbit antibodies against $\beta$-hCG (1:1,000; cat. AP13036b, Abgent, San Diego, CA, USA), EMT markers $\beta$-catenin, Slug, vimentin, Snail, claudin, N-cadherin and E-cadherin (1;1,000; cat. 9782; Cell Signaling Technology). Membranes were washed in Tris-buffered saline with Tween-20 (TBST) and peroxidase-conjugated AffiniPure goat anti-rabbit IgG (1:10,000; cat. KGAA35; KeyGen Biotech) and peroxidaseconjugated goat anti-mouse IgG secondary antibody (1:10,000; cat. L3032-2; Signalway Antibody, College Park, $\mathrm{MD}$, USA) were added and incubated at room temperature for $1 \mathrm{~h}$. The membranes were washed with PBST three times, and visualization of the protein bands was achieved using an enhanced chemiluminescence Plus kit (cat. WBKLS0500; Millipore, Billerica, MA, USA) as recommended by the manufacturer.
Construction of a peritoneal xenograft model in nude mice. All animal procedures were approved by the Institutional Use and Care of Animals Committee. Female nude mice, aged 4-6 weeks (weighing $20 \mathrm{~g}$ ) were housed and cared for at the Animal Center of Tongji University (Shanghai, China). Abdominopelvic cavity invasion is the most common form of tumor dissemination in human ovarian cancer in the clinic; therefore, direct intraperitoneal implantation in mice is a routine method to simulate an ovarian cancer model to measure metastatic ability in vivo. For xenografts, $1 \times 10^{7}$ cells transfected with LV-vector or LV- $\beta$-hCG were intraperitoneally injected into mice (6 mice/group) in the right flank. Xenograft growth was monitored by NightOWL LB 983 In Vivo Imaging System (cat. LB983 NC100; Berthold Technologies, Bad Wildbad, Germany) every two days. Mice were sacrificed after 20 days of follow-up, or according to tumor burden. Tumors were removed, fixed in $10 \%$ formalin, and subjected to routine histological examination. The ovaries, uterus, omentum, spleen, liver, intestines, colon and kidney were dissected from the mice, fixed in $10 \%$ formalin and IHC stained to detect $\beta$-hCG. All mouse experiments were conducted according to the approved animal protocol of the Animal Center of Tongji University.

Statistical analysis. All experiments were repeated at least three times in duplicates. Data are presented as the mean values \pm SEM. The data were tested for significance employing Student's unpaired t-test, Chi-square test and analysis of variance (ANOVA). The level of significance was set at $\mathrm{P}<0.05$.

\section{Results}

$\beta$-hCG is highly elevated in metastatic tissue compared to tumor tissue of the ovary and normal tissue. To determine the biological function of $\beta$-hCG in human ovarian cancer progression, IHC was performed to examine $\beta$-hCG expression in 20 normal ovarian tissue samples, 24 human ovarian cancer tissue samples and 24 metastatic tissues of ovarian cancer samples. The location of the metastatic sample were mainly from omentum and mesenterium, and all the tumor samples used in the present study were epithelial ovarian tumors, including serous ovarian and mucinous ovarian cancer, and clear cell ovarian carcinoma, verified and analyzed by two gynecologic pathologists. Results showed that $5.0 \%(1 / 20), 54.2 \%(13 / 24)$ and $83.3 \%(20 / 24)$ of the cases were $\beta$-hCG-positive in normal ovarian tissue samples, human ovarian cancer tissue samples and metastatic tissue of ovarian cancer samples, respectively. $\beta$-hCG was expressed at significant levels in the metastatic tissues of ovarian cancer and at relatively insignificant levels in the primary ovarian cancer tissues, and it was barely expressed in normal ovarian tissue $(\mathrm{P}<0.05)($ Fig. 1$)$, which suggested that $\beta$-hCG may play an important role in tumorigenesis and metastasis of ovarian cancer.

Successful construction of $\beta$-hCG-overexpressing and silenced ovarian cancer cell lines. To obtain a $\beta$-hCG overexpression model in ovarian cancer cells, LV- $\beta$-hCG or the LV-vector (both with a GFP gene) were introduced into the ovarian cancer cell lines ES-2 and SKOV3 via a lentiviral 
A

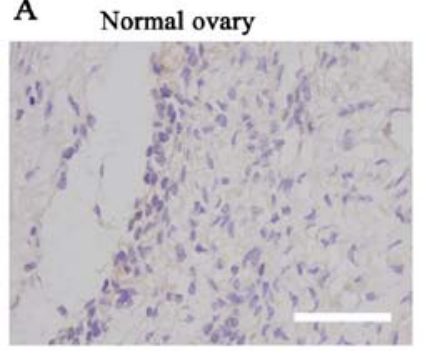

EOC

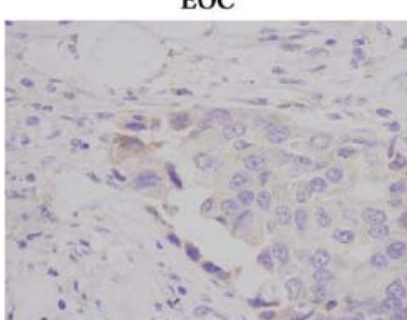

Metastasis of EOC

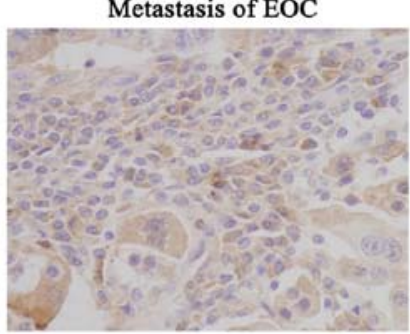

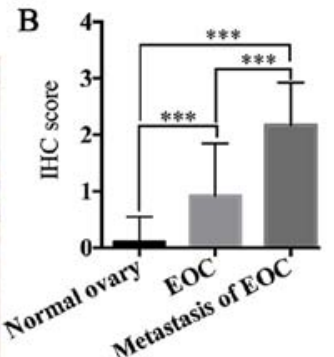

Figure 1. $\beta$-hCG is highly elevated in metastatic tissues of ovarian cancer compared to ovarian cancer and normal ovarian tissues. (A) Representative immunohistochemical staining for $\beta$-hCG in normal ovarian tissue, ovarian cancer tissue and metastatic tissue of ovarian cancer revealed the highest levels of $\beta$-hCG in metastatic tissue of ovarian cancer. Scale bars, $50 \mu \mathrm{m}$. (B) Average scores of representative immunohistochemical staining for $\beta$-hCG in normal ovarian tissue, ovarian cancer tissue and metastatic tissue of ovarian cancer were quantified $\left({ }^{* * *} \mathrm{P}<0.001\right)$.
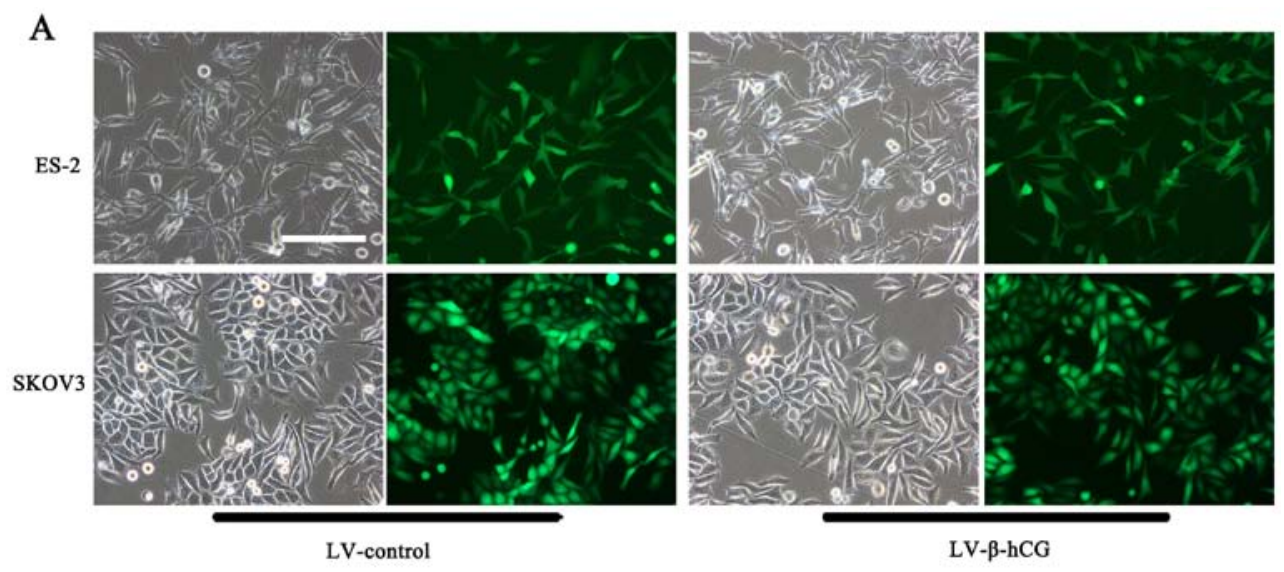

B

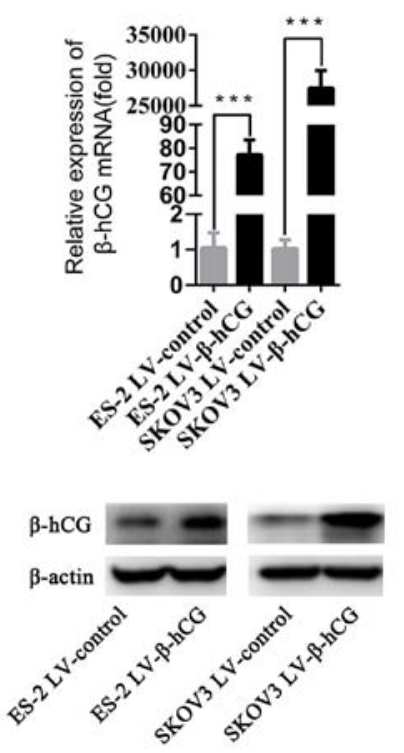

C

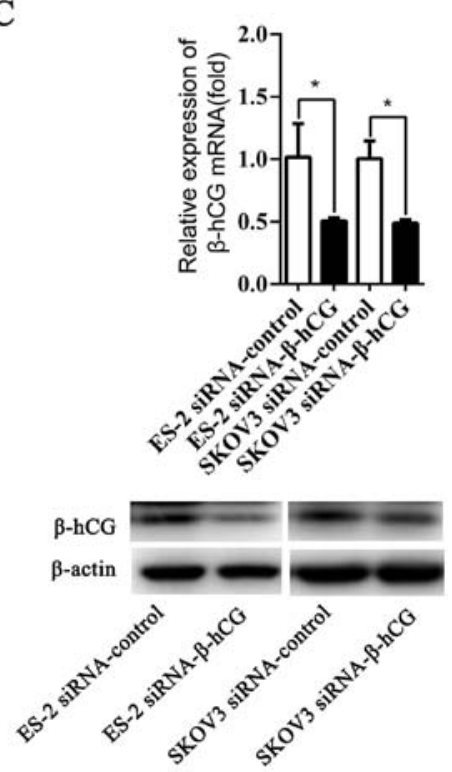

Figure 2. Successful construction of $\beta$-hCG-overexpressing and -silenced ovarian cancer cell lines. (A) ES-2 and SKOV3 cells were transfected with LV-vector or LV- $\beta$-hCG. Cells were photographed under a fluorescence microscope, using both light and fluorescence. Scale bar, $200 \mu \mathrm{m}$. (B) The relative expression level of $\beta$-hCG mRNA in $\beta$-hCG-overexpressing ES-2 and SKOV3 cells according to RT-PCR analysis and western blotting (**** $\mathrm{P}<0.001)$. (C) The relative expression level of $\beta$-hCG mRNA in $\beta$-hCG-silenced ES-2 and SKOV3 cells was determined by RT-PCR analysis and western blotting ("P<0.05).

expression vector. Sequence information for the LV- $\beta$-hCG and LV-vector plasmids were validated by sequencing (data not shown). Stable $\beta$-hCG-overexpressing cell lines were selected with puromycin. As shown in Fig. 2A, the stable cell lines were successfully constructed, and nearly 90-95\% infection efficiency was determined by GFP assay in both ES-2 and SKOV3 cell lines using fluorescence microscopy. Results showed that $\beta$-hCG was obviously overexpressed at both the mRNA and protein levels in the LV- $\beta$-hCG-transfected ES-2 and SKOV3 cells compared with the control group by RT-PCR and western blotting $(\mathrm{P}<0.001)$ (Fig. $2 \mathrm{~B})$. In addition to construction of stable overexpressing $\beta$-hCG cell lines, depletion of endogenous expression of $\beta$-hCG in ES-2 and SKOV3 cells was also established through transient transfection with a 


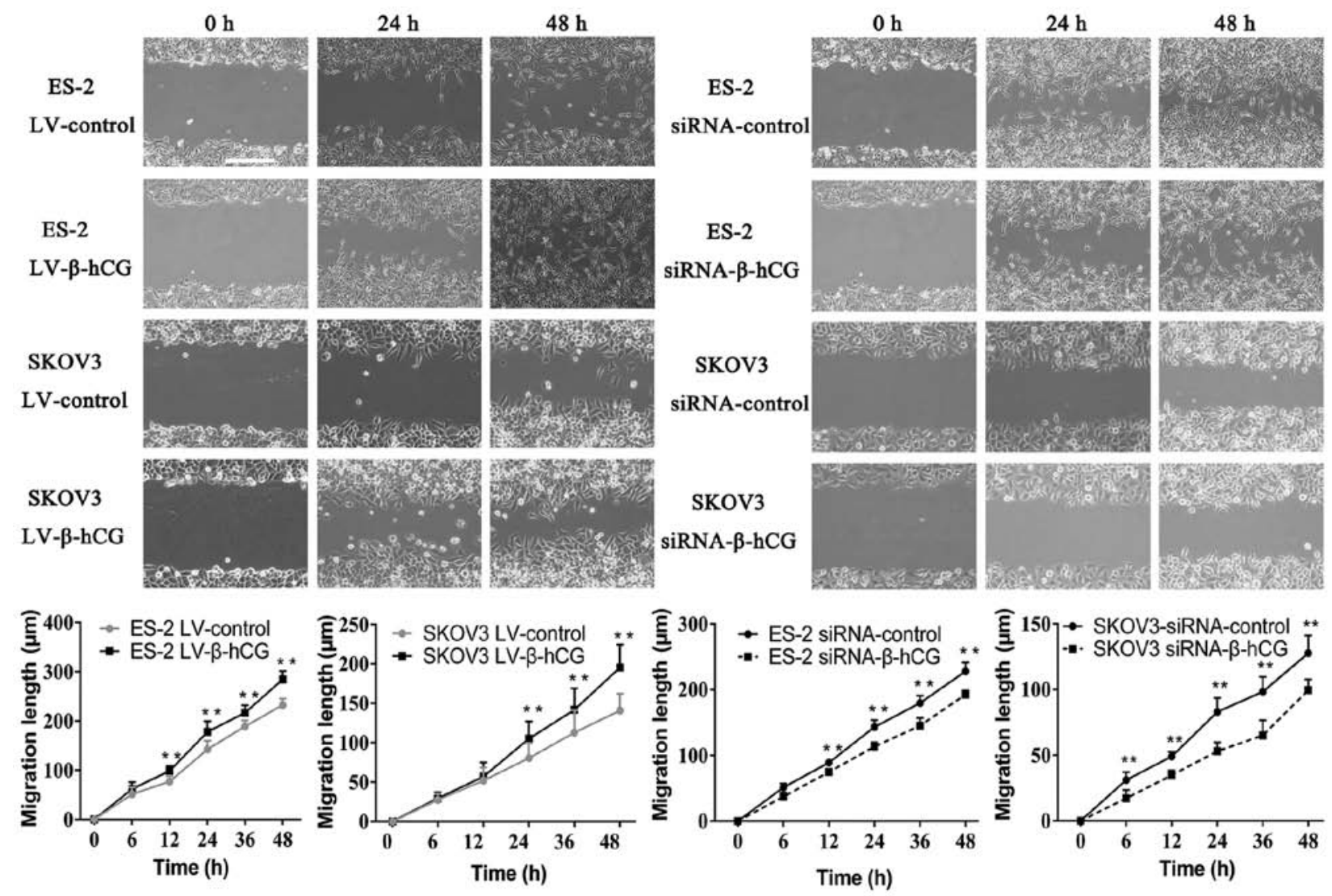

Figure 3. Wound-healing assay showed that $\beta$-hCG regulates ovarian cancer cell migration in vitro $\left({ }^{* *} \mathrm{P}<0.01\right)$.

small interference RNA (siRNA) technique and was confirmed by RT-PCR and western blotting $(\mathrm{P}<0.05)$ (Fig. 2C).

$\beta$ - $h C G$ regulates ovarian cancer cell migration, morphology and attachment ability in vitro. Wound-healing and Transwell assays were performed to validate the effect of $\beta$-hCG overexpressing and silencing of ovarian cancer ES-2 and SKOV3 cells on migration and invasion ability. As shown in Figs. 3 and 4 the overexpression of $\beta$-hCG greatly increased cell migration and invasion abilities compared with the control $(\mathrm{P}<0.001)$, while the inhibition of $\beta$-hCG expression resulted in a slower rate to fill the gap in the wound-healing assay, and the number of cells that penetrated through the Matrigel matrix was decreased in the Transwell invasion assay, which indicated that inhibition of $\beta$-hCG markedly decreased migration and invasion abilities in the ES-2 and SKOV3 cells $(\mathrm{P}<0.01)$. To investigate the biological changes induced by $\beta$-hCG expression, a colony-formation assay was performed to assess alterations in cell morphology. Under microscopic observation, the stable $\beta$-hCG-overexpressing ES- 2 and SKOV3 cells displayed an elongated spindle-like mesenchymal morphology and became dissociated from each other, whereas control cells exhibited a cobblestone-like epithelial phenotype (Fig. 5A). In addition, a cell adhesion assay was performed to investigate the adhesive ability of ovarian cancer cells mediated by $\beta$-hCG expression. The results showed that the adhesion proportion was significantly decreased in the $\beta$-hCG-overexpressing ES-2 and SKOV3 cells, while adhesion proportion was highly increased in the $\beta$-hCG-seilenced cells, which indicated that
$\beta$-hCG markedly regulated ovarian cancer cell attachment ability $(\mathrm{P}<0.05)$ (Fig. 5B).

$\beta$-hCG regulates EMT in ovarian cancer cells. To determine the potential molecular mechanisms of $\beta$-hCG in EMT, western blot analysis was performed to detect the expression of epithelial and mesenchymal protein markers. The results showed that overexpression of $\beta$-hCG upregulated the expression of mesenchymal markers: vimentin, $\mathrm{N}$-cadherin, $\beta$-catenin, Slug and Snail, while it downregulated the expression of the epithelial markers E-cadherin and claudin (Fig. 6A). Conversely, the $\beta$-hCG-depleted ES- 2 and SKOV3 cells demonstrated increased E-cadherin and claudin expression, but decreased vimentin, $N$-cadherin, $\beta$-catenin, Slug and Snail expression. Furthermore, the immunofluorescence staining of $\beta$-hCG-overexpressing cells pictured by laser scanning confocal microscopy were consistent with the western blot results (Fig. 6B).

Overexpression of $\beta$ - $h$ CG promotes metastasis in a nude mouse peritoneal xenograft tumor model. $\beta$-hCG-overexpressing and control cells were intraperitoneally injected into nude mice, and the growth of xenografts was continuously detected by NightOWL LB 983 In Vivo Imaging System. Results showed that $\beta$-hCG upregulation promoted tumor burden and spread range (Fig. 7A). On the 23rd and 26th day after intraperitoneal inoculation, ES-2 and SKOV3 group nude mice were sacrificed and dissected, respectively. Gross visualization showed that overexpression of $\beta$-hCG induced more and larger metastatic 

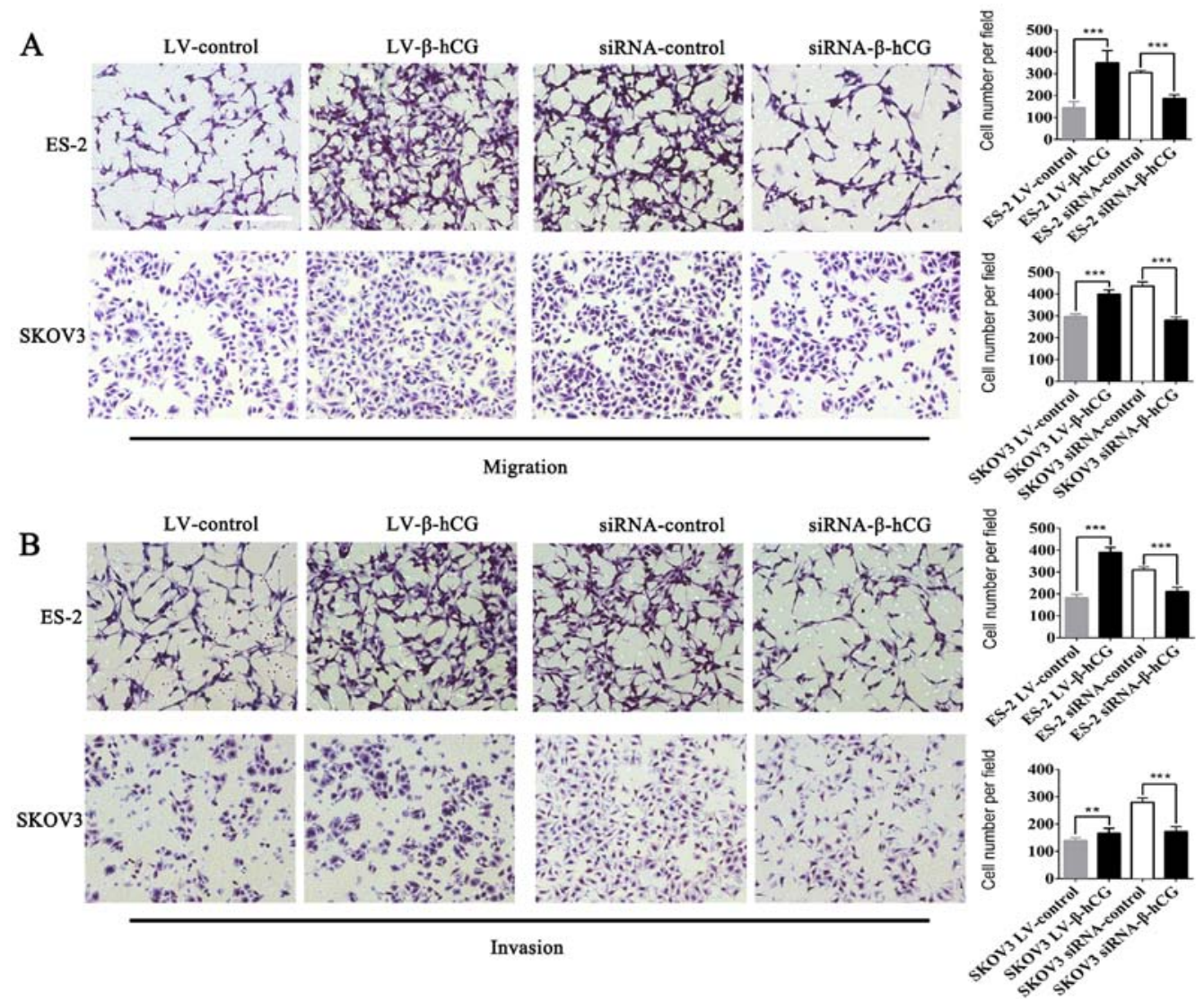

Figure 4. Transwell assay showed that $\beta$-hCG regulates ovarian cancer cell migration $(\mathrm{A})$ and invasion $(\mathrm{B})$ in vitro $\left({ }^{* *} \mathrm{P}<0.01,{ }^{* * *} \mathrm{P}<0.001\right)$.

A

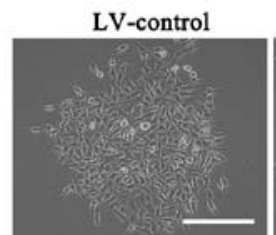

LV- $\beta$-hCG
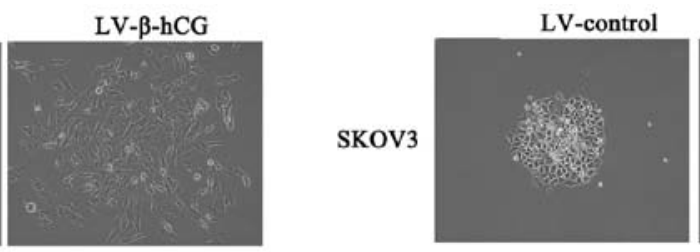

B
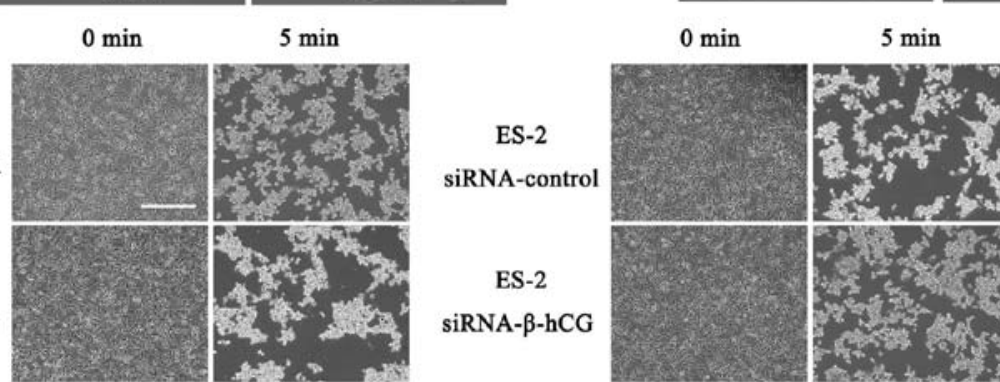

20 minute
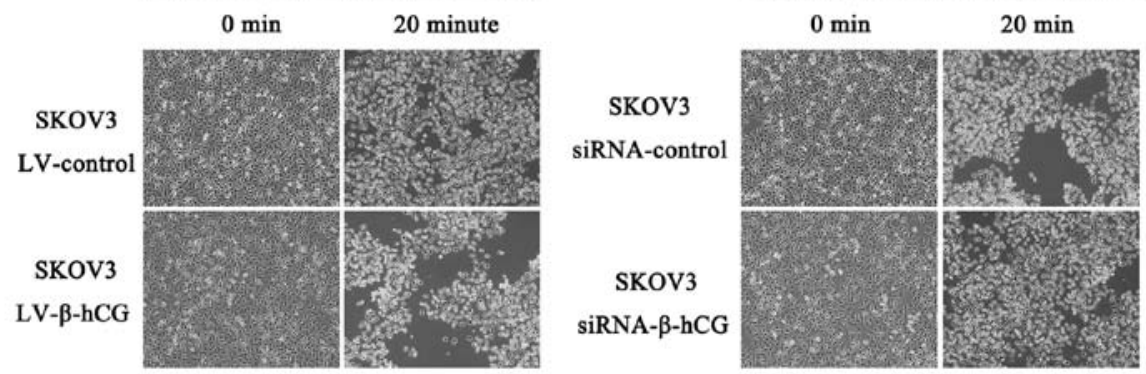

LV- $\beta$-hCG
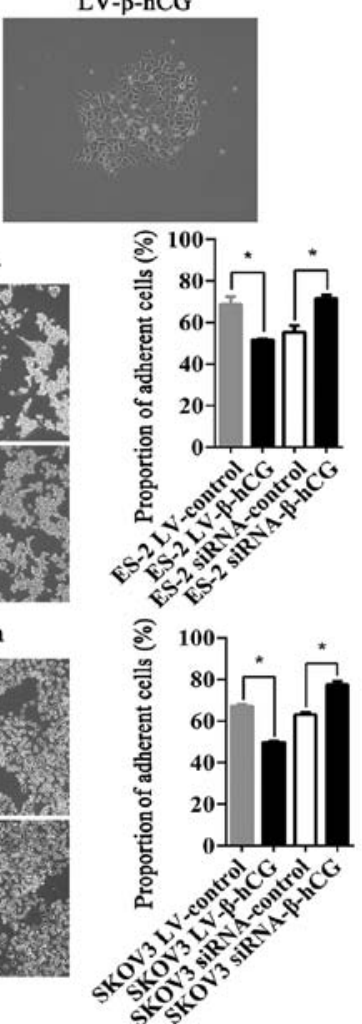

Figure 5. $\beta$-hCG regulates ovarian cancer cell morphology and attachment ability in vitro. (A) Morphology of epithelial ovarian cancer cells mediated by $\beta$-hCG expression. The cell phenotype changed from an epithelial morphology to a spindle-like mesenchymal morphology after $\beta$-hCG upregulation. Scale bar, $200 \mu \mathrm{m}$. (B) Cell adhesion assays showed that ES-2 and SKOV3 cells transfected with the $\beta$-hCG vector had more loose adhesion ability than cells treated with the control. Scale bar, $200 \mu \mathrm{m}\left({ }^{*} \mathrm{P}<0.05\right)$. 
A
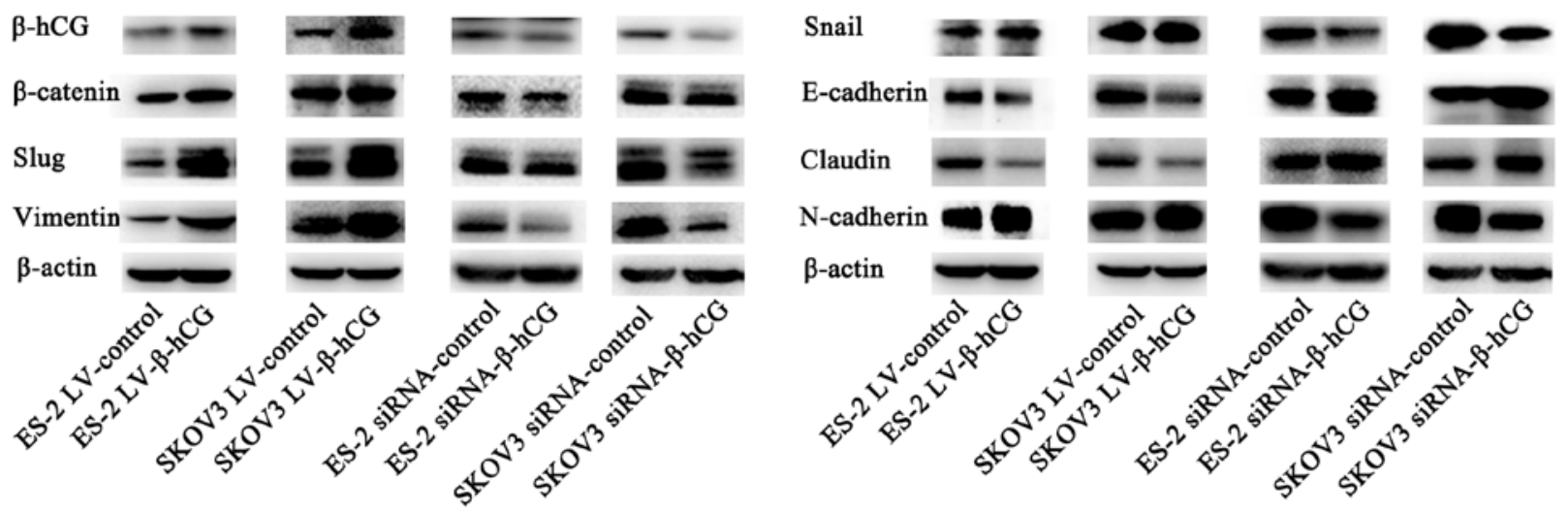

B
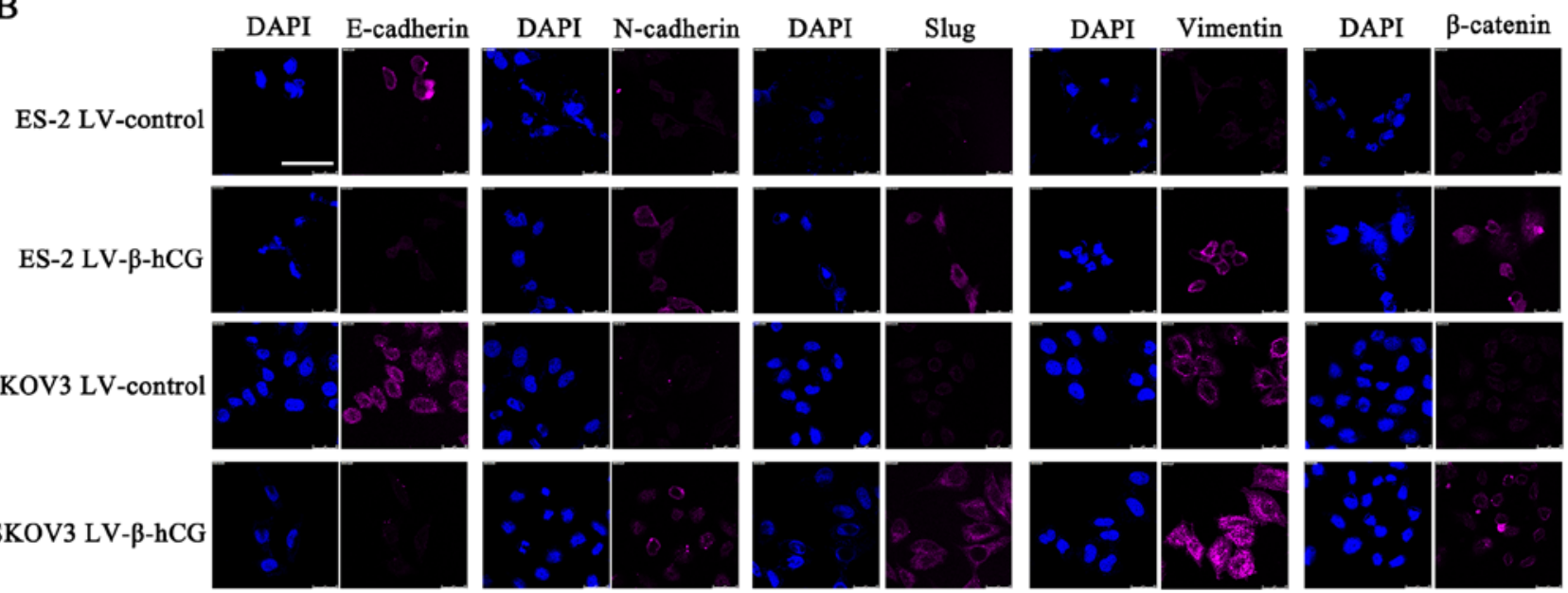

Figure 6. $\beta$-hCG induces EMT in ovarian cancer cells. (A) Expression levels of EMT markers in ES-2 and SKOV3 cells transfected with different reagents, as determined by western blotting. (B) Immunofluorescence staining of EMT markers in $\beta$-hCG-overexpressing and control cells pictured by laser scanning confocal microscopy. Scale bar, $50 \mu \mathrm{m}$.

nodule formation in omentum, mesentery, peritoneum and the diaphragm compared with the control group (Fig. 7B). In order to objectively evaluate the metastasis ability regulated by $\beta$-hCG, all the metastatic nodules, diameter $>2 \mathrm{~mm}$, were collected and weighted, and the results showed that $\beta$-hCG significantly promoted tumor metastasis (Fig. 7C). Pathology experts confirmed nodular lesions observed with the naked eye to be ovarian cancer tissue by microscopic examination of $\mathrm{H} \& \mathrm{E}$-stained tissues, and $\beta$-hCG was confirmed to be upregulated compared to the control group by IHC (Fig. 7D).

\section{Discussion}

HCG is an accurate marker that is currently widely used in clinical diagnosis and monitoring of pregnancy, trophoblastic and ovarian germ cell tumors. Of course, the involvement of hCG in the progression of malignancy is intrinsically different from pregnancy-associated hCG. Among the five family members of hCG, sulfated hCG and hCG are hormones produced by placental syncytiotrophoblast cells and pituitary gonadotrope cells, whereas hyperglycosylated hCG is an autocrine factor produced by placental cytotrophoblast cells, which drives malignancy in placental cancers and testicular and ovarian germ cell malignancies. $\beta$-hCG and hyperglycosylated $\beta$-hCG are autocrine factors produced by many advanced malignancies (4).
We found that $\beta$-hCG facilitated proliferation and tumorigenesis in ovarian epithelial cells and was significantly elevated in malignant ovarian tumors, compared with normal epithelial expression in ovaries, fallopian tubes and endometrium (11). In the present study, we observed that the expression of $\beta$-hCG in metastases was obviously higher compared to the tumor tissues from the ovary, which indicates that $\beta$-hCG may play a role in promoting the spread of ovarian cancer. To verify this speculation, $\beta$-hCG was upregulated and downregulated in ovarian cancer cell lines by lentiviral transfection and siRNA interference techniques, respectively, and the modulated effect of $\beta$-hCG on migration and invasion of ovarian cancer cells was confirmed by a series of experiments in vitro and in vivo.

Some scholars have found that $\beta$-hCG may promote ovarian cancer growth and vasculogenic mimicry formation via activation of the luteinizing hormone receptor signal transduction pathway (12). Various scholars have demonstrated that $\beta$-hCG, Erk1/2 and MMP-2 are potential targets with which to block glioblastoma invasion (13). There are other scholars that have verified that $\beta$-hCG has a cystine knot structure, and this structure happens to be similar to that of transforming growth factor $\beta$ (TGF $\beta$ ), and thus, can seemingly antagonize a TGF $\beta$ receptor, while the pregnancy-related hormone $\mathrm{hCG}$ does not appear to expose these sequences and structures $(14,15)$.

TGF- $\beta$, a ubiquitously expressed cytokine, is perhaps the best-characterized promoter of EMT (16). EMT refers to a 
A
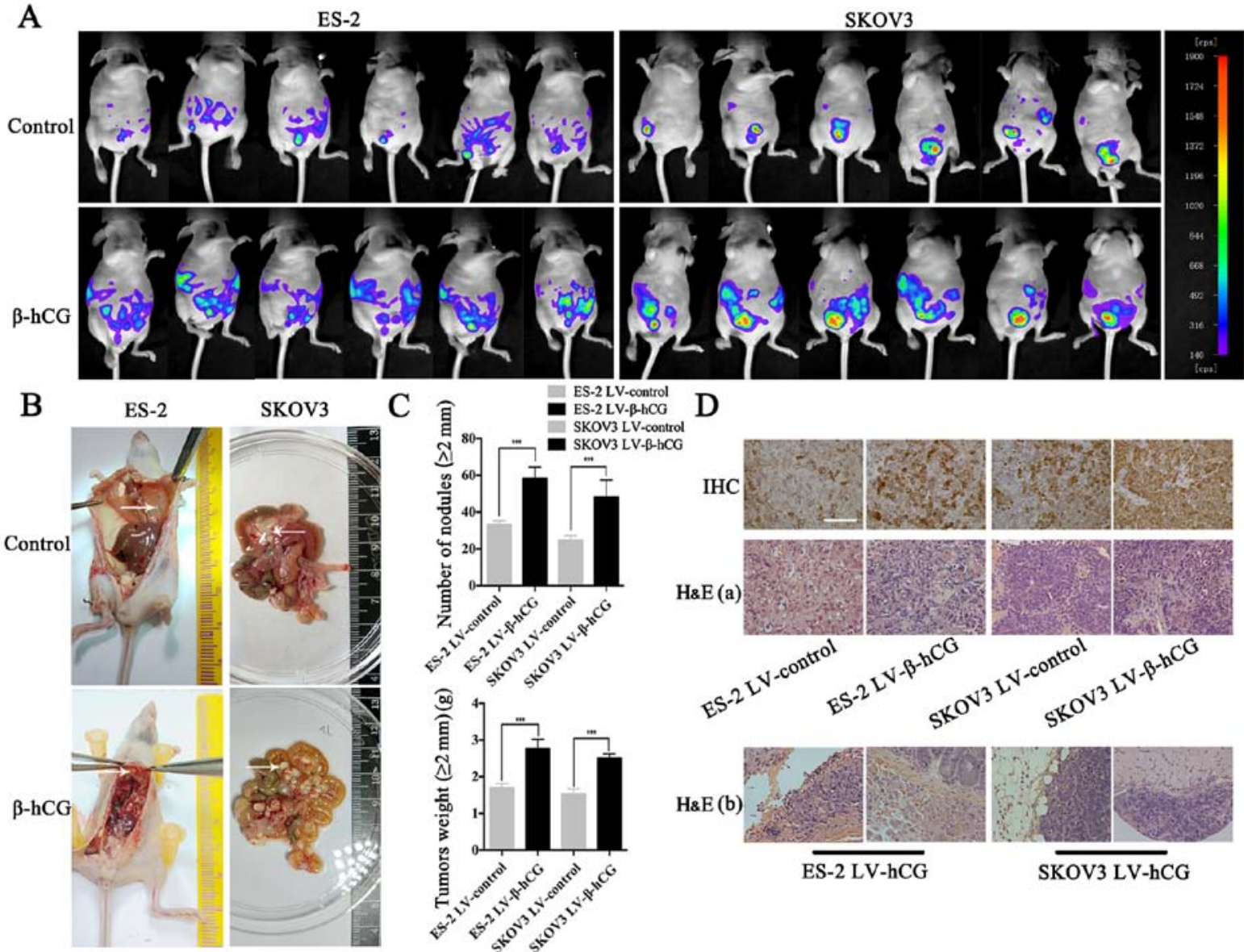

\section{rol}
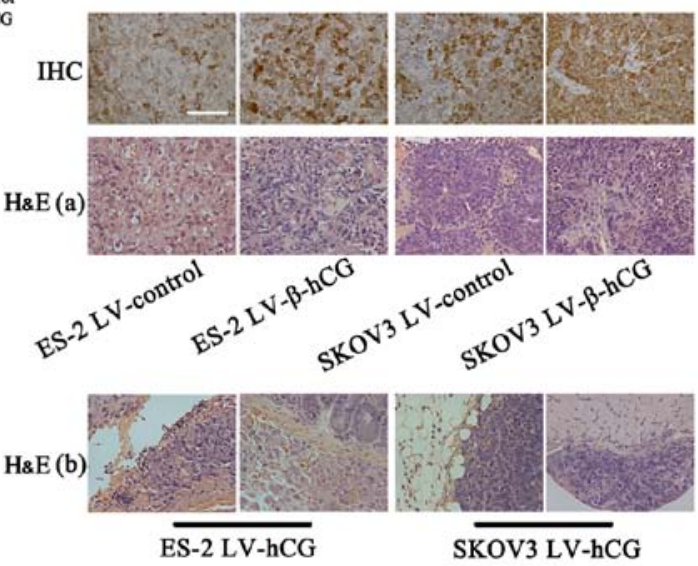

Figure 7. Overexpression of $\beta$-hCG promotes metastasis in a nude mouse peritoneal xenograft tumor model. (A) The growth of xenografts was detected by NightOWL LB 983 in vivo imaging system. (B) After being sacrificed, dissemination of peritoneal tumor in nude mice was assessed, and the significant difference in metastasis in mesentery and diaphragm are marked by white arrows. (C) Average number of the peritoneal tumor nodules, and average tumor weight of each group were quantified $\left({ }^{* * *} \mathrm{P}<0.001\right)$. (D) Representative images of IHC for $\beta$-hCG and H\&E in xenograft tumor tissues. H\&E (a) The cell morphological characteristics of the xenografts by H\&E staining were observed under a microscope. H\&E (b) The cell morphological of metastatic lesions in nude mice with -overexpressed $\beta$-hCG by H\&E staining, from left to right as follows: the mesentery and small intestine metastases of the ES-2 group, and the omental and diaphragmatic metastases of the SKOV3 group. Scale bars, $50 \mu \mathrm{m}$.

global cellular and molecular transition by which polarized epithelial cells gain mesenchymal properties allowing them to migrate, which plays a vital role in local invasion and metastatic dissemination during malignancy (17). Activating TGF $\beta$ ligands initiate signaling, and closely related Smaddependent pathways, including phosphoinositide 3-kinase (PI3K)-Akt (18), focal adhesion kinase (FAK) (19), p38 mitogen-activated protein kinase (p38 MAPK) (20), and extracellular signal-regulated kinase (Erk) (21), have been identified as crucial for EMT. During EMT, epithelial cells reorganize their cytoskeleton, resolve cell-cell junctions and switch off the expression of epithelial markers, turning on mesenchymal genes, such as, E-cadherin, vimentin, $\mathrm{N}$-cadherin, $\beta$-catenin, Snail, claudin, ZO-1 and others (22). Accordingly, we found that overexpression of $\beta$-hCG induced morphological changes, and the appearance of epithelial ovarian cancer cells changed from closely arranged polygons into a fusiform morphology with a loose arrangement, demonstrating that $\beta$-hCG has a role in regulating EMT-associated gene expression.

In combination with the findings from other recent studies, our results shed light on the molecular mechanisms of $\beta-\mathrm{hCG}$ expression and its functional role in promoting ovarian carcinoma metastasis and invasion, and thus may point to a new target for therapeutic intervention.

\section{Acknowledgements}

The present study was supported by the National Natural Science foundation of China (no. 81372305).

\section{References}

1. Siegel RL, Miller KD and Jemal A: Cancer statistics, 2016. CA Cancer J Clin 66: 7-30, 2016.

2. Jelovac D and Armstrong DK: Recent progress in the diagnosis and treatment of ovarian cancer. CA Cancer J Clin 61: 183-203, 2011.

3. Bachmayr-Heyda A, Auer K, Sukhbaatar N, Aust S, Deycmar S, Reiner AT, Polterauer S, Dekan S and Pils D: Small RNAs and the competing endogenous RNA network in high grade serous ovarian cancer tumor spread. Oncotarget 7: 39640-39653, 2016.

4. Cole LA: HCG variants, the growth factors which drive human malignancies. Am J Cancer Res 2: 22-35, 2012.

5. Khare P, Bose A, Singh P, Singh S, Javed S, Jain SK, Singh O and Pal R: Gonadotropin and tumorigenesis: Direct and indirect effects on inflammatory and immunosuppressive mediators and invasion. Mol Carcinog 56: 359-370, 2017. 
6. Lempiäinen A, Stenman UH, Blomqvist $\mathrm{C}$ and Hotakainen $\mathrm{K}$ : Free beta-subunit of human chorionic gonadotropin in serum is a diagnostically sensitive marker of seminomatous testicular cancer. Clin Chem 54: 1840-1843, 2008.

7. Jankowska AG, Andrusiewicz M, Fischer N and Warchol PJ: Expression of $h C G$ and $G n R H s$ and their receptors in endometrial carcinoma and hyperplasia. Int J Gynecol Cancer 20: 92-101, 2010

8. Muller CY and Cole LA: The quagmire of hCG and hCG testing in gynecologic oncology. Gynecol Oncol 112: 663-672, 2009.

9. Hameed A, Miller DS, Muller CY, Coleman RL and AlboresSaavedra J: Frequent expression of beta-human chorionic gonadotropin (beta-hCG) in squamous cell carcinoma of the cervix. Int J Gynecol Pathol 18: 381-386, 1999.

10. Mustafa A, Bozdag Z, Tepe NB and Ozcan HC: An unexpected reason for elevated human chorionic gonadotropin in a young woman. Cervical squamous carcinoma. Saudi Med J 37: 905-907, 2016.

11. Guo X, Liu G, Schauer IG, Yang G, Mercado-Uribe I, Yang F, Zhang S, He Y and Liu J: Overexpression of the $\beta$ subunit of human chorionic gonadotropin promotes the transformation of human ovarian epithelial cells and ovarian tumorigenesis. Am J Pathol 179: 1385-1393, 2011.

12. Gao S, Fan C, Huang H, Zhu C, Su M and Zhang Y: Effects of HCG on human epithelial ovarian cancer vasculogenic mimicry formation in vivo. Oncol Lett 12: 459-466, 2016.

13. Li Z, Du L, Li C and Wu W: Human chorionic gonadotropin $\beta$ induces cell motility via ERK1/2 and MMP-2 activation in human glioblastoma U87MG cells. J Neurooncol 111: 237-244, 2013.

14. Tegoni M, Spinelli S, Verhoeyen M, Davis P and Cambillau C: Crystal structure of a ternary complex between human chorionic gonadotropin (hCG) and two Fv fragments specific for the alpha and beta-subunits. J Mol Biol 289: 1375-1385, 1999.
15. Cole LA and Butler S: Hyperglycosylated hCG, hCG $\beta$ and Hyperglycosylated hCG $\beta$ : Interchangeable cancer promoters. Mol Cell Endocrinol 349: 232-238, 2012.

16. O'Connor JW and Gomez EW: Biomechanics of TGF $\beta$-induced epithelial-mesenchymal transition: Implications for fibrosis and cancer. Clin Transl Med 3: 23-35, 2014.

17. Jung HY, Fattet L and Yang J: Molecular pathways: Linking tumor microenvironment to epithelial-mesenchymal transition in metastasis. Clin Cancer Res 21: 962-968, 2015.

18. Bakin AV, Tomlinson AK, Bhowmick NA, Moses HL and Arteaga CL: Phosphatidylinositol 3-kinase function is required for transforming growth factor beta-mediated epithelial to mesenchymal transition and cell migration. J Biol Chem 275: 36803-36810, 2000.

19. Cicchini C, Laudadio I, Citarella F, Corazzari M, Steindler C, Conigliaro A, Fantoni A, Amicone L and Tripodi M: TGFbetainduced EMT requires focal adhesion kinase (FAK) signaling. Exp Cell Res 314: 143-152, 2008.

20. Bhowmick NA, Zent R, Ghiassi M, McDonnell M and Moses HL: Integrin beta 1 signaling is necessary for transforming growth factor-beta activation of p38MAPK and epithelial plasticity. J Biol Chem 276: 46707-46713, 2001.

21. Xie L,Law BK, Chytil AM,Brown KA, Aakre ME and Moses HL: Activation of the Erk pathway is required for TGF-beta1-induced EMT in vitro. Neoplasia 6: 603-610, 2004.

22. Kalluri R and Weinberg RA: The basics of epithelial-mesenchymal transition. J Clin Invest 119: 1420-1428, 2009. 